\title{
Tuberöse Sklerose
}

\section{Das Gleich gewicht im mTOR-Signalweg wieder herstellen}

- Tuberöse Sklerose (TSC) ist eine seltene, genetisch bedingte komplexe Systemerkrankung, bei der der mTOR-Signalweg aufgrund eines Gen-Defektes überaktiviert ist. Die Folgen sind Fehlbildungen und gutartige Tumoren (Hamartome) in nahezu allen Organen. Dazu gehören etwa subependymale Riesenzellastrozytome (SEGA), die sich bei bis zu $20 \%$ der TSC-Patienten entwickeln und zu einem Hydrozephalus, neurologischen Ausfällen bis hin zum Tod führen können.

Bis vor kurzem war die chirurgische Resektion die einzige Therapieoption bei TSC SEGA. Diese kann jedoch mit Komplikationen verbunden sein, da die Tumoren tief im Gehirn lokalisiert sind. Seit September 2011 ist Everolimus (Votubia ${ }^{\circledR}$ ) für die Behandlung von TSC-Patienten ab drei Jahren mit SEGA zugelassen, die eine therapeutische Maßnahme benötigen, für die aber eine Operation nicht angemessen ist. Der mTOR-Inhibitor kann das gestörte Gleichgewicht im mTOR-Signalweg wieder herstellen und so Tumorzellwachstum-, -stoffwechsel und -angiogenese bei TSC-Patienten wirkungsvoll hemmen.

\section{SEGA-Volumen bei allen Patienten reduziert}

Basis der Zulassung von Everolimus bei TSC SEGA sind die Ergebnisse einer prospektiven, einarmigen, offenen Phase-II-Studie, an der 28, im Mittel elf Jahre alte TSCPatienten mit seriellem SEGA-Wachstum teilnahmen [Krueger DA et al. N Engl J Med 2010; 363: 1801-11]. Die Patienten erhielten über sechs Monate eine Monotherapie mit Everolimus (initial $3 \mathrm{mg} / \mathrm{m}^{2}$ Körperoberfläche bei einem angestrebten Blutspiegel von 5-15 ng/ $\mathrm{ml}$ ). Primärer Endpunkt war die Reduktion des SEGA-
Volumens in diesem Zeitraum. 75\% der Patienten erreichten eine Verringerung des SEGA-Volumens um mindestens $30 \%$ und $32 \%$ der Patienten um mindestens $50 \%(\mathrm{p}<0,001)$. In allen 28 Fällen ging das Volumen der SEGA zurück [Franz DN et al. Vortrag ASCO Annual Meeting 2010]. Während der Behandlung musste bezüglich der SEGA keine Operation erfolgen und es traten keine neuen Läsionen auf. Auch kam es weder zu einer Verschlechterung noch zur Neubildung eines Hydrozephalus und es gab keine Anzeichen für erhöhten Hirndruck. Weiterhin wurde eine Besserung faszialer Angiofibrome und eine signifikante Reduktion epileptischer Anfälle $(p=0,02)$ beobachtet. Eine weitere Auswertung während der zeitlich unbegrenzten Extensionsphase der Studie zeigte, dass die Wirksamkeit von Everolimus auch nach einer medianen Therapiedauer von etwa drei Jahren erhalten blieb [Krueger DA et al. Poster Summit on drug discovery in TSC and related disorders 2011].

Everolimus wurde in der Studie einschließlich der Extensionsphase gut vertragen. Es traten keine Medikamenten-bedingten Grad-4-Nebenwirkungen auf und keiner der Patienten brach die Therapie aufgrund von Nebenwirkungen ab. Häufigste Medikamenten-bedingte Nebenwirkungen waren Stomatitiden, Infektionen der oberen Atemwege und Sinusitiden, die überwiegend nur gering ausgeprägt waren (Grad 1/2). red

\section{STEPS - besser leben mit Schizophrenie und bipolaren Störungen}

Das Unternehmen Otsuka Pharma GmbH hat das Service-Programm „STEPS - Schritt für Schritt besser leben" entwickelt, das Patienten mit Schizophrenie und bipolaren Störungen bei der Krankheitsbewältigung im Alltag unterstützen soll.

STEPS basiert auf dem Konzept der individuellen Patientenansprache und bietet dem Teilnehmer hilfreiche Informationen sowie praktische Tipps rund um die jeweilige Erkrankung und thematisiert zudem die häufigsten Probleme, die im Alltag auftreten können. Mit dem Ziel die Compliance zu verbessern und den Patienten zur aktiven Krankheitsbewältigung zu motivieren, erhält jeder Teilnehmer des Programms individuell auf ihn zugeschnittene Informationen. Diese bestehen aus praktischen Tipps rund um die jeweilige Erkrankung, den Alltag sowie mögliche Therapieformen. Je nach Wunsch werden sie per neutraler Post nach Hause oder per E-Mail an den Patienten versendet. Der behandelnde Arzt ist immer über den Einschluss des Patienten in das Programm informiert, weiß daher über alle Maßnahmen Bescheid und bekommt zusätzlich Materialien für seine Patientengespräche zur Verfügung gestellt.

Weitere Informationen sowie zusätzliche Downloads und eine Smartphone-App unter: www.meine-steps.de Weitere Auskünfte auch unter 069170086115 oder unter E-Mail: info@meine-steps.de. 